\title{
Blockchain Technology as a Health and Safety Contributor in the Transport and Logistics Industry - Human Resource Requirements
}

\author{
Richard Skiba \\ LRES Training Management \\ Melbourne, Australia
}

\begin{abstract}
Adaptation of blockchain technology in the Transport and Logistics industry may provide for not only increased transparency, trust and efficiency but also improved safety by minimising mis-declaration of cargo. There are currently a number of operators in the industry developing applications based on blockchain with may others planning to utilise the approach. As organisations commit to blockchain based projects, there will be an increased demand for blockchain developers, and associated job roles, and this paper considers the skills and knowledge requirements for those in these roles and provides a basis for determining the competence required for blockchain development.
\end{abstract}

Keywords:- Transport and Logistics; Blockchain; Smart Contract; Competency Standards; Training, Safety.

\section{INTRODUCTION}

Edwards (2019) describes that a "blockchain is a database where you can add information, but not remove it". Edwards suggests that the data stored on a blockchain can be anything, including cryptocurrency, insurance claims or even shares of physical property such as real estate. The type of data stored in a blockchain is not limited in the same way as a database is capable of storing for any number of needs or purposes. Traditionally, data is stored on a single server however in a blockchain, the data is stored on a network of computers referred to as nodes. The data is spread amongst these nodes. As such, the system is distributed, given that it exists over multiple nodes, and that there is no central point of failure such as can occur in traditional database systems maintained on a single server. Further, given that transaction data on the blocks is immutable in that it cannot be altered or forged. This makes the transaction information highly trustworthy and instills a high degree of confidence in users. The nodes work together in a shared ledger system to add new information to the blockchain in bundles that are referred to as blocks. Each time a new block of information is added, it is chained to the previous one in a linear fashion resulting in the blockchain.
The blockchain essentially removes the need for intermediaries required to act as trusted third parties to verify, record and coordinate transactions as this is achieved by effecively 'sharing' databases between multiple parties utilizing the blockchain. Gockel, Acar and Forster (2018) note that "by facilitating the move from a centralized to a decentralized and distributed system, blockchain effectively liberates data that was previously kept in safeguarded silos". As such, there is no one single entity with the power to change the state of the blockchain ledger and do things like shut down accounts or seize funds (Edwards, 2019). Edwards also outlines that when parties make a transaction via the blockchain, they only need to place trust in the underlying infrastructure of the blockchain rather than in each other. This is on the basis that a decentralised computer network can record and verify each transaction as well as make the transaction transparent, trust is created by default.

A consensus system is used in the blockchain as a fundamental aspect of the blockchain's security. This means there must be agreement between the nodes in the decentralized peer-to-peer system on the blockchain's true state and where if one node tries to lie, then its record of the blockchain won't match that of the other nodes, and in turn it will be ignored and not added to the blockchain (Edwards, 2019). This system of consensus mechanisms, where nodes are sychronised to each other. is a fundamental component to the security of the blockchain where transactions are legitimized prior to being added to the blockchain.

Sultan, Ruhi and Lakhani (2018) note that "a blockchain can be applied in virtually any industry in which assets are managed and transactions occur. It can provide a secure chain of custody for both digital and physical assets through its functional characteristics that facilitate transactions through trust, consensus, security, and smart contracts". Blockchain applications are limitless and the technology will be used in many industries, including but not limited to finance, digital identity, government, energy, healthcare, international trade and commodities, law, media and entertainment, real estate, sports, and social impact. 


\section{APPLICATIONS IN TRANSPORT AND LOGISTICS}

Global supply chains can utilise this technology and Edwards (2019) outlines that significant industry companies, such as Maersk, DHL, Amazon, and Walmart, to identify a few, are all exploring approaches to improve their existing systems utilising blockchain technology. A number of researchers, such as, cited in Tijan, Aksentijevi, Ivani and Jardas (2019), including Hackius and Petersen (2017), Rauch, Seidenstricker, Dallasega and Hämmerl (2016).and Petersen, Hackius and; Kersten (2016), have outlined the uses and benefits of blockchain technologies in logistics and supply chain management.

Gockel, et al. (2108) presents that blockchain technology can help alleviate many of the frictions in global trade logistics including procurement, transportation management, track and trace, customs collaboration, and trade finance. Some of these frictions are outlined by Vilas (2020) who informs that according to Accenture, ten percent of freight invoices are problematic including duplication, wrong freight mode changes, and incorrect fees. Vilas suggests that one solution to the ever-growing list of invoices and other pieces of certification required in transport and logistics is the blockchain improving communication and understanding between supply chain partners. This is due to information stored on the blockchain, rather than across a multitude of documents and systems. Kewalram (2019a) notes that just one shipment can generate approximately 200 communications documents, and the associated cost of processing and administering this documentation makes up an estimated 20 percent of the cost of transporting goods. Vilas (2020) posits that the transparent nature of the blockchain can remove the necessity to send a purchase order of materials, as the other party will know how much inventory the supplier has, what service level is required and the rate they will consume the material.

The blockchain can also utilise "smart contracts" for and these contracts can detect infractions in the supply chain and prevent the record being entered. This feature removes the need for a party to investigate what went wrong and how much is owed (Vilas, 2020). This is a further benefit that can be provided to the transport and logistics industry through more effective electronic systems.

Kewalram puts the use of blockchain and smart contract technologies into context with an example describing the shipping an item from one country to another as follows:

"Take the example of a sweater traveling from a factory in China to a shop in the U.K. This journey may involve hundreds of paper documents and hours of manual data entry at different (and multiple) points in the supply chain for the same transaction/shipment. This increases the risk of error or an incomplete data chain and a reduction of trust in the data. Often, the retailer will have to make payment in advance.

In a blockchain-enabled supply chain, this sweater could be embedded with an intelligent chip (a function of IoT), which would send a digital signal when the sweater leaves the factory. This signal would be recorded in the blockchain, and the retailer's system could automatically pay for the product as the result of a smart contract being triggered. An automated customs entry could then be lodged, which would be the equivalent to the importer making a certified declaration. Customs officials could automatically pull all the information they need from the database, knowing that nobody in the process has tampered with the data. When the sweater arrives, the sender could instantly verify that the right person has received it through identity management that is blockchain-enabled" (Kewalram, 2019a).

Kewalram (2019a) outlines another significant benefit in blockchain use in transport and logistics in that it can help improve shipping industry safety. According to data from the Cargo Incident Notification System, "close to a quarter of all serious incidents on containerships are attributable to misdeclared cargo" (Kewalram, 2019a). Dangerous cargo that has been incorrectly or inaccurately identified can potentially lead to serious incidents that can include fires and other accidents. This in turn, can lead to substantial losses, damage and delays, with the potential to cause significant harm or fatality. The Company of Master Mariners Australia (2020) estimates that a major container ship fire occurs at sea 60 days on average and that "that across the intermodal spectrum as a whole, $66 \%$ of incidents related to cargo damage can be attributed to poor practice in the overall packing process; that is not just in securing but also in cargo identification, declaration, documentation and effective data transfer". An identified critical aspect here being the correct declaration and handling of dangerous goods and this is often skewed in manual documentation systems.

Company of Master Mariners Australia (2020) utilises information from published government inspections to note that $20 \%$ of containers are poorly packed or incorrectly identified, which translates into 1.3 million potentially unstable dangerous goods containers traveling around the world each year. On this basis alone, an improved approach, such as offered through a blockchain approach, is validated. The aim of blockchain based data management, in this case, being to generate more transparency and accountability in tracking dangerous goods; ultimately, reducing incidents. Forster (2020) concurs noting that "shipping dangerous goods relies on effective and reliable exchange of information throughout the chain - from shipper to carrier to receiver and all those in between or on the side lines". Forster recognises that current methods are slow, inefficient and prone to errors and are further complicated if the cargo changes hands during shipment. Forster (2020) contextualises the use of blockchain technology to address these problems with the following example: 
"Let's look at how this could apply to carrying dangerous goods cargoes. A recognised ploy of some shippers is to declare the cargo as non-dangerous at time of booking but then amend it at the very last minute to declare that it is in fact a dangerous goods cargo.

The shipper hopes that the changes are not processed in time and the carrier fails to be informed at loading, therefore carrying the cargo as if it were non-dangerous. But using a system based on blockchain, the more timely and transparent exchange of information could result in the carrier being better positioned to make the necessary changes and compliance arrangements".

\section{IMPLEMENTATION IN TRANSPORT AND LOGISTICS}

In order to implement blockchain approaches, Pareek (2020) recognises that as a first step, logistics companies are required to educate themselves, and then digitize, standardize, and cleanse their data. Kewalram (2019b) informs that "until a critical mass of new industry participants fully understands the benefits of frictionless international trade, embraces the potential of the new technology and creates effective forums for joint implementation, the promise of more efficient, streamlined trade around this technology will be slow to be realized". Blockchain technology has not yet been integrated into the transport and logistic industry although there are current moves toward this direction. In order to further do so, Kewalram (2019b) notes that organizations need to ensure they recruit or build the right skills in their organizations and make the exploration and implementation of technology like blockchain one of their key strategic priorities.

One of the most prominent activities in the implementation of blockchain technology in the industry is by Maritime Blockchain Labs (MBL). They have formed a consortium to explore the use of blockchain to address the misdeclaration and handling of dangerous goods using digital tools to improve the tracking of dangerous goods cargo, as well as online audit trails for transparency and accountability in locating dangerous goods and reducing incidents. The consortium, including Copenhagen Malmö Ports (CMP), Flexport, X-Press Feeders, SecureSystem, DSV, Port+, Agility and MTI are developing a prototype to evaluate the possibility of using distributed ledger technology to address the problems that are faced by stakeholders throughout the supply chain.
Daley (2019) describes a number of blockchain logistics projects currently being implemented. As examples, Chronicled, a blockchain-based network developer, combines blockchain with Artificial Intelligence and Internet of Things (IoT) devices to automate traceability and instantaneously approve financial transactions in the shipping industry. ShipChain in California built a blockchain based logistics platform to support the shipping process from end-to-end. The developed platform allows all parties on the chain to receive updated information on a container's location, as well as estimated arrival times, via encrypted public ledgers. In Hong Kong, 300cubits' tokens are used as cryptocurrency for the logistics world. Using smart contracts and the company's TEU tokens, suppliers can ensure that they will provide a certain quantity of products to ship and shipping companies are able to set deadlines and quality standards for their shipping processes. Koopman Logistics, in the Netherlands, is an automotive transportation company utilises blockchain to ship cars globally. By utilising the ledger technology, they have quickened the payment process and made the shipping process more secure. Other companies such as CargoLedger in the Netherlands, Slync and Blockfreight in California and Fr8 Network in New York, build blockchain-based software for the logistics industry.

Although there are a number of projects across the industry that are targeted at the whole supply chain, projects and applications could be applied at an organisational level across a range of transport and logistics operations. A trucking company, for example can benefit from using Internet of Things and blockchain in trucks, to monitor all sorts of information regarding the cargo in order to do a proper cost-profit analysis. Organisations can likewise utilise smart contracts to speed up the payment process, automatically triggering payment upon delivery verification where the simple act of scanning the barcode on a box or receiving an electronic signature will trigger that payment.

Blockchain technology implementation at an organisational level requires a range of skills and capabilities within the workplace, which generally are not currently available within those industries. Australian Industry Standards (2020) acknowledges that the implementation of blockchain technology will require the workforce to be equipped with the right skills to perform their tasks, helping to maximise efficiency and ensure compliance through blockchain development. 
Human resource development is required across various layers of an organisation. Levy (2020) suggests that there are two key groups: Leaders and Managers and Software Developers. The first group, leaders and managers, need to become well versed in blockchain technology to help leverage this new technology into their organisations. The second group, software developers, need to learn how to program for blockchain, in particular, using smart contracts. A software developer undertaking blockchain development, develops smart contracts and web applications, and is responsible for developing and optimizing blockchain rules and procedures and constructing the architecture of blockchain system.

A blockchain developer's knowledge and skill are not specific to a particular industry, such as transport and logistics, but rather categorised as information technology or software development competence that can be applied to any industry. These skills and knowledge include, as outlined by the Sharma (2019), data structures, blockchain architecture, smart contract development, web development and cryptography. Sharma advises that blockchain "makes use of a plethora of data structures along with advanced cryptography for building a secure and immutable system". It is important for blockchain developers to understand the data structures properly.

Smart contracts refer to self-executing contracts in that contain the terms of the agreement between the buyer and the seller and these are written into lines of programming code. Developers need to understand all aspects of smart contract development, including learning network-specific programming languages such as Viper, Chaincode and Solidity (Sharma, 2019). Further, internet application development is a fundamental aspect that a blockchain developer must learn. A blockchain developer must be proficient in both front-end and back-end development including API handling, request handling, and creating interactive graphical user interfaces for decentralised applications (DApps).

Finally, blockchain developers need to have a good grasp of cryptography given the use of "cryptographic methods such as hash functions like SHA256 and KECCAK256 apart from asynchronous cryptography for generating digital signatures" (Sharma, 2019). Aside from the technical development skills and knowledge, the developers will also need the skills to work with the industry stakeholders to determine their needs, and the needs specific to the industry, and ensure the developed products meet those needs.

Apart from blockchain developers, there are a number of related job roles emerging as the technology increases in its adaptation. Mearian (2018) considers and describes some of these, one of which is the role of Blockchain Project Manager. These project managers convert an organisation's needs in common English into technical language to the extent that specifications can be developed, and then from the blockchain developers' language back into regular English, such that the technology can be used by the organisation. Blockchain Quality Engineers are also required and they ensure quality in all areas of blockchain development, including automation frameworks and tests, manual testing and dashboards.

All blockchain structures fall into three categories, being either a public blockchain architecture, private blockchain architecture or consortium blockchain architecture. Lastovetska (2019) notes that a public blockchain architecture means that the data and access to the system is available to anyone who is willing to participate, a private blockchain architecture is controlled only by users from a specific organization or authorized users who have an invitation for participation. Aa consortium blockchain architecture, on the other hand, can consist of a few organizations with procedures established and controlled by the preliminary assigned users. Organisations select a blockchain architecture that suits their needs or application.

\section{BLOCKCHAIN DEVELOPER SKILLS AND KNOWLEDGE}

Blockchain Developers must be able to write in a native programming language without the use of any frameworks given that much of the role's activity is essentially a programming exercise. Effective programming skills include a knowledge of the basics of engineering and development, problem analysis and problem-solving and these skills form part of the core skills of a competent blockchain developer. Programming skills include proficiency in a language such as $\mathrm{C}++$, Java, Python, NodeJS, and/or C \#, for example. Developers must also be able to compare blockchain systems, their algorithms and protocols and analyse the main types of system vulnerabilities such that they are able to protect the system from attacks (Fironov, 2020). Aside from these skills, web development skills are required, which may include a knowledge of HTML, CSS, NodeJS, Angular, and MongoDB.

The complexity of blockchain requires a high level knowledge of data structures and their application. At the core, a distributed ledger is like a network of replicated databases, only it stores information in blocks rather than tables. The blocks are also cryptographically secured to ensure their integrity every time a block is added. Blockchain developers, as such, need knowledge of how common data structures, such as binary search trees, hash maps, graphs, and linked lists, work and the ability to build these. Knowledge and application of data structures should be developed concurrently with programming language development. 
A smart contract is a program that runs on the blockchain once a transaction is complete to enhance blockchain's capabilities. Mugo (2019) notes that smart contracts become more difficult to secure as the increase in their complexity, and as such, blockchain developers need to be aware of every possible way a smart contract can be executed and manipulated and ensure that it does what is expected. This encompasses acquiring the skills and knowledge to optimize and audit smart contracts.

Mougayar (2016) categorised ten areas of development required for understanding in blockchain development. These include: Cryptocurrency; Computing Infrastructure; Transaction Platform; Decentralized Database; Distributed Accounting Ledger; Development Platform; Open Source Software; Financial Services Marketplace; Peer-to-Peer Network; and, Trust Services Layer. Each of these should be included in the development of any competency standard related to blockchain development as they represent the core required knowledge and skills.

Performance elements, then, should include:

$>$ Engage with client to define and specify the business problem to be investigated

$>$ Review current organisational documentation

$>$ Review the technical requirements

$>$ Use a range of information gathering techniques to define requirements and approaches

$>$ Examine and explain Real-World Use Cases of Blockchain Technology

$>$ Confirm business critical factors relating to current and future directions with stakeholders

Establish system boundaries and scope

$>$ Select blockchain type suitable to the application

$>$ Select and obtain the appropriate languages and technology to meet the development requirements

$>$ Develop plans to implement proposed changes according to organisational policy and procedures

$>$ Use object-oriented programming languages for blockchain application development

$>$ Develop Ethereum smart contracts

$>$ Develop DApps

$>$ Demonstrate appropriate blockchain data safeguarding techniques

$>$ Develop application web backend

$>$ Create interface pages using the appropriate languages

$>$ Ensure that new system requirements are documented incorporating relevant standards

$>$ Develop technical documentation

$>$ Gather and analyse feedback
Required skills should include:

access and analyse relevant information on changes to technology and resources

$>$ develop anf formalise client requirements

$>$ conduct interviews with client and staff

$>$ write code for popular blockchain programming platforms, including Hyperledger and Ethereum

$>$ develop code utilising pre-existing and user-defined data structures

$>$ develop code utilising standard algorithms

$>$ select and use blockchain architectures

$>$ perform Blockchain network security risk analysis

$>$ utilise web development programming and techniques

$>$ design and develop technical documentation, including procedures, training material and user guides

$>$ Incorporate relevant standards for documentation and coding as required

Required knowledge could include:

$>$ Analysis methods and planning approaches to technical problems

$>$ Project management processes

$>$ Attributes of public vs private blockchain

$>$ Blockchain transaction process

$>$ Block creation

$>$ Using Digital Signatures

$>$ Programming language structure and syntax for a range of languages such as C++, Javascript, Python, Solidity

$>$ Programming modularity, extensibility, and security

$>$ Produce code utilising user-defined data structures and applying standard algorithms

$>$ Code debugging techniques

$>$ Code testing methods

$>$ Producing technical documentation of activities and completed application

$>$ Ethereum and Smart Contract application development techniques

$>$ Blockchain cryptography

$>$ DApp Frameworks

$>$ Design principles for DApp developers

$>$ Building serverless applications

$>$ Using blockchain consensus mechanisms - Proof of work (PoW), proof of stake $(\mathrm{PoS})$, delegated proof of stake (DPoS), proof of burn, proof of important (PoI), proof of elapsed time (PoET)

$>$ Applying best security practices for Blockchain System

$>$ Using HTML, CSS, NodeJS, Angular, and MongoDB for blockchain applications

> Limitations of blockchain technology 
Corresponding to the competency elements outlined above, Hikido (2020) confirm these requirements in their specification for a blockchain developer by means of a job description, stating:

"Typical tasks for a Blockchain Developer Include:

Create architecture features and interfaces by using programming languages

$>$ Research and design blockchain technologies

$>$ Develop and test blockchain technologies

$>$ Protecting the application from bugs and cyber attacks.

$>$ Working closely with engineers, sales personnel, and other information technology employees in designing and creating innovative solutions.

$>$ Research create and document existing and new blockchain structures and proposed solutions

$>$ Writing excellent multithreaded codes

$>$ Analyzing the needs,resources, and problems of the company and provide necessary solutions

$>$ Working with open-source projects and codebases

$>$ Keeping up with current blockchain technology and cryptocurrency trends

Technical Skills for Blockchain Developers:

$>$ Strong background in software development, computer science or engineering.

$>$ Strong programming skills

$>$ Knowledge of Java \& C++

$>$ Understanding other programming languages, like Python, STL, C++11,JavaScript-node.js.

$>$ Strong back-end skills

$>$ Algorithms \& data structures

$>$ Basic knowledge of cryptography

$>$ Peer-to-peer or P2P networks

$>$ Bitcoin-like blockchains

$>$ Qt Widgets module for GUI wallet application

$>$ Knowledge of current technology tools like microservice architectures and Docker containers"

\section{CONCLUSION}

In addition to technical design and development skills required by blockchain developers, there is a broad need for experts in legal and compliance requirements, and for those who understand the impact of a decentralized approach to data management and analysis, in order to implement blockchain approaches in the transport and logistics industry. For these roles, and those of blockchain developers, in many cases these job roles have not yet been defined and there is a general lack of competency standards around what those work roles entail. In order for blockchain development to occur, there are core skills, knowledge and performance requirements that blockchain developers require to competently develop applications for industry. There is an emerging need for blockchain based applications in the transport and logistics industry and once developed, these will lead to improved safety and efficiency and also result in greater transparency, security and traceability.

\section{REFERENCES}

[1]. Australian Industry Standards. (2020). TRANSPORT AND LOGISTICS IRC: Annual Update to Industry Skills Forecast and Proposed Schedule of Work2020. Retrieved

from https://www.australianindustrystandards.org.au/wpcontent/uploads/2020/03/TLI-SF-MERGEDDRAFT.pdf.

[2]. Company of Master Mariners Australia. (2020). A major containership fire occurs every 60 days. Retrieved from http://www.mastermariners.org.au/newsinternational/3350-a-major-containership-fire-occursevery-60-days.

[3]. Daley, S. (2019). Making moves: How blockchain is quickly becoming a must-have in logistics. Retrieved from https://builtin.com/blockchain/blockchainsupply-chain-logistics-uses.

[4]. Edwards, J. (2019). What is blockchain technology? How blockchain works, who's using it and why it's much more than just bitcoin. Retrieved from https://www.finder.com.au/blockchain-guide.

[5]. Fironov, G. (2020). Blockchain Developer Skill Set. Retrieved from https://hakin9.org/blockchaindeveloper-skill-set/.

[6]. Forster, A. (2020). The frequency of container fires has been a long-standing concern in the shipping industry. $\quad$ Retrieved from https://www.nepia.com/articles/using-tech-to-tackledangerous-goods-cargoes/.

[7]. Gockel, B., Acar, T., \& Forster, M. (2018). Blockchain in Logistics: Perspectives on the upcoming impact of blockchain technology and use cases for the logistics industry. DHL Customer Solutions \& Innovation: Troisdorf, Germany.

[8]. Hackius, N.; Petersen, M. (2017). Blockchain in Logistics and Supply Chain: Trick or Treat. Retrieved from

https://tubdok.tub.tuhh.de/bitstream/11420/1447/1/pet ersen_hackius_blockchain_in_scm_and_logistics_hicl _2017.pdf.

[9]. Hikido. (2020). How to Write an Blockchain Developer Job Description. Retrieved from http://blog.hikido.com/blockchain-developer-jobdescription/.

[10]. Kewalram, B. (2019a). Blockchain Could Revolutionize Logistics, But Is The Industry Prepared To Let It? Retrieved from https://www.forbes.com/sites/forbestechcouncil/2019/ 12/04/blockchain-could-revolutionize-logistics-but-isthe-industry-prepared-to-let-it/\#2b508db811e2.

[11]. Kewalram, B. (2019b). Blockchain: Is the Shipping Industry Ready? Retrieved from https://www.agility.com/insights/future-oflogistics/blockchain-is-the-shipping-industry-ready/.

[12]. Lastovetska, A. (2019). Blockchain Architecture Basics: Components, Structure, Benefits \& Creation. Retrieved from https://mlsdev.com/blog/156-how-tobuild-your-own-blockchain-architecture. 
[13]. Levy, G. (2020). The skills your employees need to implement blockchain applications. Retrieved from https://www.udemy.com/blog/dont-get-disruptedblockchain-skills-your-business-needs-for-success/.

[14]. Mearian, L. (2018). The top blockchain jobs you need to know about. Retrieved from https://www.computerworld.com/article/3277617/thetop-blockchain-jobs-you-need-to-know-about.html.

[15]. Mougayar, W. (2016). The business blockchain: promise, practice, and application of the next Internettechnology. John Wiley \& Sons, Inc., Hoboken, New Jersey

[16]. Mugo, J. (2019). What it takes to become a blockchain developer. Retrieved from https://opensource.com/article/19/4/blockchain-careerdeveloper.

[17]. Pareek, S. (2020). How Blockchain Will Revolutionize Logistics. Cloud Credential Council. Retrieved from https://www.cloudcredential.org/blog/howblockchain-will-revolutionize-logistics/.

[18]. Petersen, M.; Hackius, N.; Kersten, W. (2016). Blockchains für produktion und logistik. ZWF, 111, 626-629.

[19]. Rauch, E.; Seidenstricker, S.; Dallasega, P.; Hämmerl, R. (2016). Collaborative Cloud Manufacturing: Design of Business Model Innovations Enabled by Cyberphysical Systems in Distributed Manufacturing Systems. J. Eng. 2016, 1-12.

[20]. Sharma, T. K. (2019). 5 Skill Sets a Blockchain Developer Must Have. Retrieved from https://www.blockchain-council.org/blockchain/5skill-sets-a-blockchain-developer-must-have/.

[21]. Karim Sultan, K.,Ruhi, U., \& Lakhani, R. (2018). Conceptualizing Blockchains: Characteristics \& Applications. 11th IADIS International Conference Information Systems.

[22]. Tijan, E., Aksentijevi, S., Ivani, K. \& Jardas, M. (2019). Blockchain Technology Implementation in Logistics. Sustainability, 11(1185.

[23]. Vilas, V. (2020). Blockchain Logistics: Blockchain Trends 2020. Retrieved from https://transporttmsandlogisticstms.com/blockchainand-logistics/. 